\title{
Quenching and Restoration of Orbital Angular Momentum Through a Dynamic Bond in a Cobalt(II) Complex
}

DOI:

10.1021/jacs.0c02257

\section{Document Version}

Accepted author manuscript

Link to publication record in Manchester Research Explorer

\section{Citation for published version (APA):}

Su, S., Wu, S., Baker, M. L., Bencok, P., Azuma, N., Miyazaki, Y., Nakano, M., Kang, S., Shiota, Y., Yoshizawa, K., Kanegawa, S., \& Sato, O. (2020). Quenching and Restoration of Orbital Angular Momentum Through a Dynamic Bond in a Cobalt(II) Complex. Journal of the American Chemical Society. https://doi.org/10.1021/jacs.0c02257

Published in:

Journal of the American Chemical Society

\section{Citing this paper}

Please note that where the full-text provided on Manchester Research Explorer is the Author Accepted Manuscript or Proof version this may differ from the final Published version. If citing, it is advised that you check and use the publisher's definitive version.

\section{General rights}

Copyright and moral rights for the publications made accessible in the Research Explorer are retained by the authors and/or other copyright owners and it is a condition of accessing publications that users recognise and abide by the legal requirements associated with these rights.

\section{Takedown policy}

If you believe that this document breaches copyright please refer to the University of Manchester's Takedown Procedures [http://man.ac.uk/04Y6Bo] or contact uml.scholarlycommunications@manchester.ac.uk providing relevant details, so we can investigate your claim.

\section{OPEN ACCESS}




\title{
Quenching and Restoration of Orbital Angular Momentum Through a Dynamic Bond in a Cobalt(II) Complex
}

\author{
Sheng-Qun Su+, Shu-Qi Wu+, Michael L. Baker"s, Peter Bencok"l, Nobuaki Azuma ${ }^{\perp}$, Yuji Miyazaki", Mo- \\ tohiro Nakano, Soonchul Kang ${ }^{\circ}$, Yoshihito Shiota', Kazunari Yoshizawa ${ }^{+}$, Shinji Kanegawa', Osamu \\ Sato*
}

'Institute for Materials Chemistry and Engineering and IRCCS, Kyushu University, 744 Motooka, Nishi-ku, Fukuoka, 8190395, Japan

The University of Manchester at Harwell, Didcot, OX11 OFA, United Kingdom

${ }^{\S}$ Department of Chemistry, The University of Manchester, Manchester M139PL, United Kingdom

"Diamond Light Source, Science Division, Didcot OX11 0DE, United Kingdom

${ }^{\perp}$ Research Core for Interdisciplinary Science, Okayama University, 3-1-1 Tsushimanaka, Kita-ku, Okayama 700-8530, Japan

${ }^{*}$ Research Center for Thermal and Entropic Science, Graduate School of Science, Osaka University, Toyonaka, Osaka, 560-

0043, Japan

${ }^{\circ}$ Graduate School of Engineering, Hiroshima University, 1-4-1 Kagamiyama, Higashi-Hiroshima, 739-8527, Japan

KEYWORDS (Word Style “BG_Keywords”). Orbital angular momentum; Dynamic bond

\begin{abstract}
Orbital angular momentum plays a vital role in various applications, especially magnetic and spintronic properties. Therefore, controlling orbital angular momentum is of paramount importance to both fundamental science and new technological applications. Many attempts have been made to modulate the ligand-field induced quenching effects of orbital angular momentum to manipulate magnetic properties. However, to date, reported changes in the magnitude of orbital angular momentum are small in both molecular and solid-state magnetic materials. Moreover, no effective methods currently exist to modulate orbital angular momentum. Here, we report a dynamic bond approach to realize a large change in orbital angular momentum. We have developed a Co(II) complex that exhibits coordination number switching between six and seven. This cooperative dynamic bond switching induces considerable modulation of the ligand field, thereby leading to substantial quenching and restoration of the orbital angular momentum. This switching mechanism is entirely different from those of spin-crossover and valence tautomeric compounds, which exhibit switching in spin multiplicity.
\end{abstract}

\section{INTRODUCTION}

Considerable effort has been expended in controlling quantum spin to develop switchable magnetic molecules,' such as ferromagnetic materials, ${ }^{2}$ spin-crossover materials, ${ }^{3,6}$ valence tautomeric complexes, ${ }^{7-15}$ and organic radicals with monomer-dimer transition, ${ }^{1-1-18}$ and, for the purpose of its application to future data-storage media, sensors and switching units. The fact that magnetic properties arise from the spin and the orbital angular momentum of electrons contained in a compound is well known. Moreover, the orbital angular momentum and spin-orbit coupling are the main physical contributions to magnetic anisotropy and play important roles in various functional materials, ${ }^{1920}$ such as molecular magnets and spintronic materials. However, in contrast to the modulation of spin angular momentum via spin-crossover transitions, controlling orbital angular momentum is in its infancy and full of challenges, due to the energetic favorability for Jahn-Teller distortions that quench orbital angular momentum. Although the presence of second order spinorbit coupling allows the partial restoration of orbital contributions to magnetization, no effective methods currently exist to comprehensively modulate the orbital angular momentum via external stimuli. Therefore, attempts to control the orbital angular momentum are scarce and the change in the orbital angular momentum as previously reported has been rather small..$^{123}$

Therefore, we propose to introduce dynamic bonds to metal complexes to realize considerable changes in the orbital angular momentum. The term "dynamic bond" represents a bond that can undergo reversible breakage and reformation with a metal center under a given external stimuli. ${ }^{24}$ The restoration of the orbital angular momentum through spin-orbit coupling is inversely proportional to the energy gap between the excited state and ground state. The subtle change of the coordination environment in the metal center usually induces only a small energy-gap change, which results in a tiny change in the orbital angular momentum as previously reported.2123 In contrast, dynamic bond formation and breakage greatly modulates the energy levels of corresponding d-orbitals. Therefore, a large change in the energy gap can be induced, and in turn, this results in a substantial change in the orbital angular momentum. ${ }^{2526}$ Using the above strategy, we focused on Co(II) and nitrate ions as the metal center and ligands, respectively. A Co(II) ion typically has a large unquenched orbital angular momentum. Moreover, 
(a)

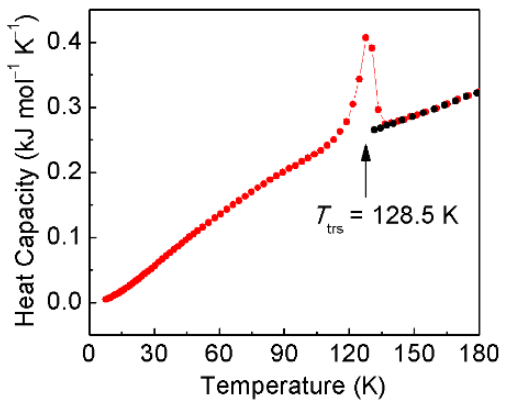

(c)

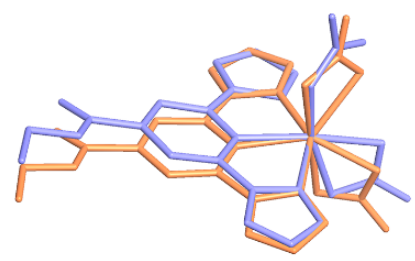

(b)
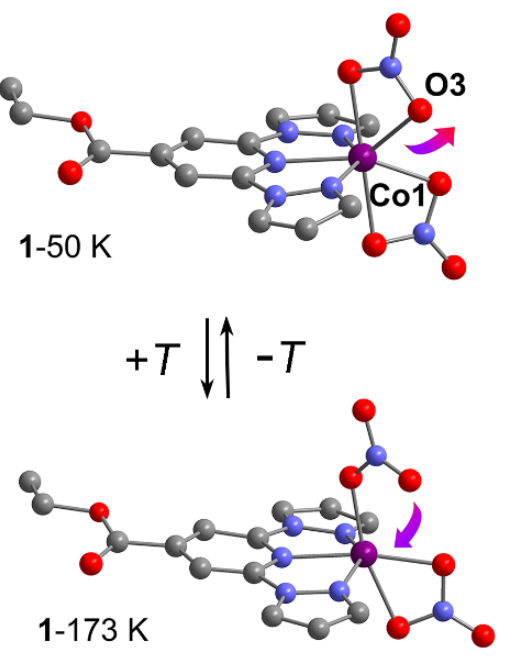
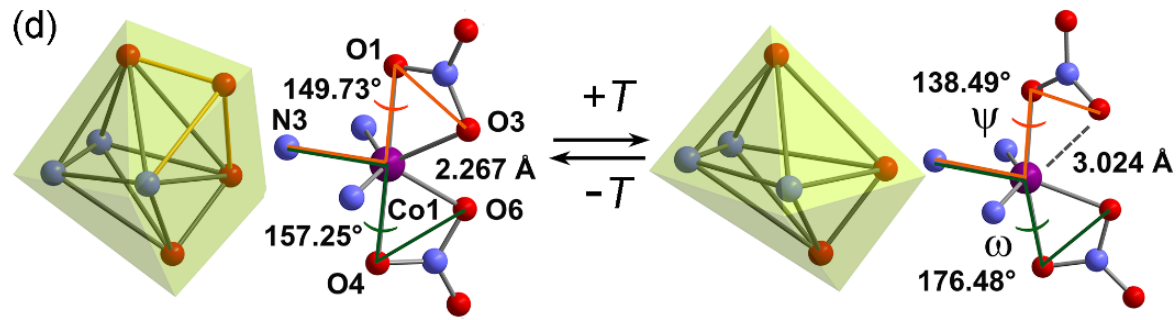

Figure 1. (a) The phase transition temperature $\left(T_{\mathrm{tr}}\right)$ is determined as $128.5 \mathrm{~K}$ upon heating after the samples cooled to $7.9 \mathrm{~K}$ (red) and 131.5 $\mathrm{K}$ (black), separately. (b) Molecular structures of crystal 1 in the low- and high-temperature phases recorded at $50 \mathrm{~K}$ and $173 \mathrm{~K}$, respectively. One of the nitrate ligands acts as a dynamic bond. (c) Molecular structure overlay directly shows the main structural changes. (d) The coordination environment changes from a seven-coordinate capped octahedral geometry to a six-coordinate octahedral geometry in the heating process attained by breaking of the coordination bond Co1-O3, which could re-form reversibly in the cooling process. The dihedral angles $\psi$ and $\omega$ also change during this process. Purple, Co; blue, N; red, O.

oxoanions such as the nitrate and carboxylate are known to adopt various coordination modes, such as monodentate or bidentate coordination, thereby potentially enabling the induction of dynamic bond behavior. ${ }^{278}$ In fact, it is well known that monodentate and bidentate coordination switching of the carboxylate ligand occurs in nonheme diiron proteins..$^{29}$

Herein, we report that a biomimetically designed cobalt complex bearing nitrate ligands exhibits magnetic bistability by changing its coordination number from six to seven. The nitrate ion undergoes reversible coordination-mode switching, which involves bonding and dissociation between $\mathrm{Co}$ (II) and an oxygen in the nitrate ion. The formation of the seventh bond modulates the ligand field causing an increase in the energy of the first singly occupied d-orbital and the breaking of the bond conversely lowers its energy, which induces effective quenching and restoration of the orbital angular momentum. The change in the magnitude of the magnetic moment induced by the modulation of the spin-orbit coupling through the dynamic bond is 10 $11 \%$ of the original magnetization value of the six-coordinate $\mathrm{Co}$ (II) compound. This change is the largest reported value for any crystalline molecular material that exhibits thermally reversible orbital angular momentum switching. The magnetization switching via this mechanism is entirely different from those of the spin-crossover and valence-tautomerism.

\section{RESULTS AND DISCUSSION}

Synthesis, structural characterization and the dynamic bond. The cobalt(II) complex, $\left[\mathrm{Co}\left(\mathrm{NO}_{3}\right)_{2}(\mathrm{~L})\right]$ (complex 1), was synthesized by layering an acetone solution of a tridentate $\mathrm{N}$ containing ligand ( $\mathrm{L}=$ ethyl-2,6-di(1H-pyrazol-1-yl)isonicotinate) on an acetone solution of $\mathrm{Co}\left(\mathrm{NO}_{3}\right)_{2} \cdot 6 \mathrm{H}_{2} \mathrm{O}$ in a glass tube; the complex was successfully obtained as purple crystals at room temperature.

The occurrence of a thermal phase transition in $\mathbf{1}$ was confirmed by heat capacity measurements using an adiabatic calorimeter (Figure 1a and Figure S1) ${ }^{30}$ The molar heat capacity under constant pressure $(\mathrm{Cp})$ showed a sharp peak accompanied by a latent heat at $128.5 \mathrm{~K}$, which is due to a phase transition, and also exhibited a super-cooling phenomenon. The change in the entropy was measured in cooling and heating modes with a hysteresis. These facts provide evidence of a first-order phase transition. The transition enthalpy and entropy estimated from the heat capacity are $\Delta \mathrm{H}=1.500 \pm 0.012 \mathrm{~kJ}$ mol-1 $^{-1}$ and $\Delta \mathrm{S}=$ $11.93 \pm 0.10 \mathrm{~J} \mathrm{~K}^{-1} \mathrm{~mol}^{-1}$, respectively.

Single-crystal XRD analysis of $\mathbf{1}$ was performed at $50 \mathrm{~K}$ (low-temperature phase, LT) and $173 \mathrm{~K}$ (high-temperature phase, HT; Figure $1 \mathrm{~b}$ and Table S1). The complex at $50 \mathrm{~K}$ crystallizes in the triclinic space group $\mathrm{P}-1$, with unit-cell parameters of $a=7.8222(16) \AA, b=8.9348(18) \AA, c=13.069(3) \AA, \alpha$ $=78.76(3)^{\circ}, \beta=73.63(3)^{\circ}, \gamma=85.45(3)^{\circ}$ and $V=859.3(4) \AA^{3}$; the asymmetric unit cell comprises one complex molecule. The cobalt ion is coordinated in a six-coordinate geometry by three nitrogen atoms of the L ligand and four oxygen atoms of two $\mathrm{NO}_{3}-$ anions $(\mathrm{O} 1, \mathrm{O} 3, \mathrm{O} 4$, and $\mathrm{O} 6)$. The two $\mathrm{NO}_{3}$ - anions act as 
terminal ligands and are located on both sides of the plane defined by the cobalt ion and the L ligand, adopting the asymmetric bidentate $\eta^{2}$-coordination mode with $\mathrm{Co}-\mathrm{O}$ distances ranging from 2.096(4) to 2.267(5) A (Figure S2a and Table S2). The above $\mathrm{Co}-\mathrm{O}$ distances fall within the usual range for cobalt compounds with nitrate ligands. The nitrate ions are twisted along the dihedral angles N3-Co1-O1-O3 $(\psi)$ and N3-Co1O4-O6 ( $\omega)$ with values of 149.73 and $157.25^{\circ}$, respectively (Figure 1d). The 3D molecular packing is primarily dictated by $\mathrm{C}-\mathrm{H} \cdots \mathrm{O}$ nonconventional hydrogen bonds, which play an important role in the cooperative phase transition and stabilization of the overall structure; the hydrogen bond distance ranges from 3.148 to $3.453 \AA$ (Figure S2c and Table S3).

When the temperature is increased, a structural change occurs. The crystal structure at $173 \mathrm{~K}$ shows that $\mathbf{1}$ has the same space group as at $50 \mathrm{~K}$ but different unit-cell parameters; the unit-cell parameters at $173 \mathrm{~K}$ are $a=7.9634(16) \AA, b=8.6405(17) \AA, c$ $=13.432(3) \AA, \alpha=82.55(3)^{\circ}, \beta=76.56(3)^{\circ}, \gamma=83.66(3)^{\circ}$ and

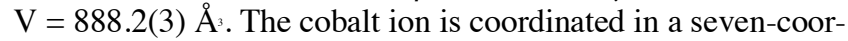
dinate geometry by three nitrogen atoms of the $\mathrm{L}$ ligand and three oxygen atoms of the two $\mathrm{NO}_{3}^{-}$anions $(\mathrm{O} 1, \mathrm{O} 4$, and $\mathrm{O} 6)$. The change in coordination geometry can be approximated as from a capped trigonal antiprismatic geometry at LT to a trigonal antiprismatic geometry at HT. The two $\mathrm{NO}_{3}$ - anions acting as terminal ligands adopt the asymmetric bidentate $\eta^{2}$-coordination mode and the monodentate $\eta$-coordination mode, respectively, with Co-O distances ranging from 2.051(2) to 2.198(2) $\AA$ (Figure S2b and Table S2). After the bond Co1-O3 breaking, the interactions between related nitrate ligands and adjacent molecules increase obviously in the $b c$ plane (Figure S2c and Table S4). The hydrogen bonds change slightly with distances ranging from 3.156 to $3.566 \AA$. Compared with the low-temperature structure, three significant changes have occurred: (1) the Co1-O3 bond $[\mathrm{Co} 1-\mathrm{O} 3=2.267$ (5)] breaks, leading to the Co1 $\cdots \mathrm{O} 3$ distance of $3.024 \AA$; (2) the dihedral angles $\psi$ and $\omega$ change to 138.49 and $176.48^{\circ}$, respectively; (3) the N3-Co1O4 angle changes from 93.25 to $109.90^{\circ}$ (Figures 1c and 1d). The breaking of the Co1-O3 bond results in a decrease in the coordination number of the cobalt center from seven to six upon heating. The calculated Mayer bond order is consistent with the breaking and forming of the Co1-O3 bond (Table S5). The data of the single-crystal XRD at $153 \mathrm{~K}$ recorded during the cooling and heating processes are almost the same, which suggests that the Co1-O3 bond can undergo reversible breakage and re-formation. For this phase transition, the number of energetically equivalent microscopic states $N$ in $\Delta \mathrm{S}=R \ln N$ is $\sim 4$ based on heat capacity measurements, where $R$ is the gas constant. The entropy originating from a vibrational mode should be considerably different between complexes with six and seven coordination numbers, which should contribute to the gain in entropy to a certain extent. However, the exact microscopic origin of the $\Delta \mathrm{S}$ remains unclear.

The above type of dynamic bond behavior can be found in nonheme diiron proteins. Interconversion between the monodentate and bidentate coordination modes of carboxylate ligands is known as a carboxylate shift, which plays a crucial role in the intimate mechanisms of catalytic reactions.$^{29}$ The experimental results for 1 show that our bio-inspired strategy to induce dynamic bonding in a crystalline compound was successful; the coordination mode of the nitrate ligand changes in response to the temperature, which is attained by the breaking and reformation of the $\mathrm{Co} 1-\mathrm{O} 3$ bond. This dynamic process was precisely characterized by single-crystal analysis, which is interesting because such precise analysis is difficult within biological molecular assemblies.

Magnetic properties. The magnetic properties of microcrystalline 1, the purity of which was confirmed through Powder Xray diffraction and elemental analysis (Figure S3), were investigated in heating and cooling modes in the temperature range $2-300 \mathrm{~K}$. The raw data were corrected for diamagnetism. The results are depicted in Fig. $2 \mathrm{a}$, where the values of $\chi_{\mathrm{M}} T$ are plotted against temperature $T$. In the temperature range $135-300 \mathrm{~K}$, the $\chi_{M} T$ value $\left(2.69 \mathrm{~cm}^{3} \mathrm{~K} \mathrm{~mol}^{-1}\right)$ depends weakly on temperature, obeying the Curie-Weiss law with a Curie constant of $\mathrm{C}=2.76$ $\mathrm{cm}^{3} \mathrm{~K} \mathrm{~mol}^{-1}$ and a Weiss constant of $\theta=-2.30 \mathrm{~K}$. Upon cooling, the $\chi_{\mathrm{M}} T$ value abruptly decreases at approximately $111 \mathrm{~K}$. The $\chi_{\mathrm{M}} T$ value at $90 \mathrm{~K}$ is $2.41 \mathrm{~cm}^{3} \mathrm{~K} \mathrm{~mol}^{--}$. The magnetic behavior at low temperature phase follows a Curie-Weiss law with $\mathrm{C}=$ $2.51 \mathrm{~cm}^{3} \mathrm{~K} \mathrm{~mol}^{-1}$ and $\theta=-2.56 \mathrm{~K}$. The decrease in $\chi_{\mathrm{M}} T$ is associated with a structural phase transition accompanied with the formation of a $\mathrm{Co}-\mathrm{O}$ bond at the molecular level. Note that the significant decrease in the $\chi_{\mathrm{M}} T$ value below $20 \mathrm{~K}$ is due to the effect of zero-field splitting. Both $\chi_{\mathrm{M}} T$ values are between the values of high-spin (HS) cobalt(II) complexes with a fully
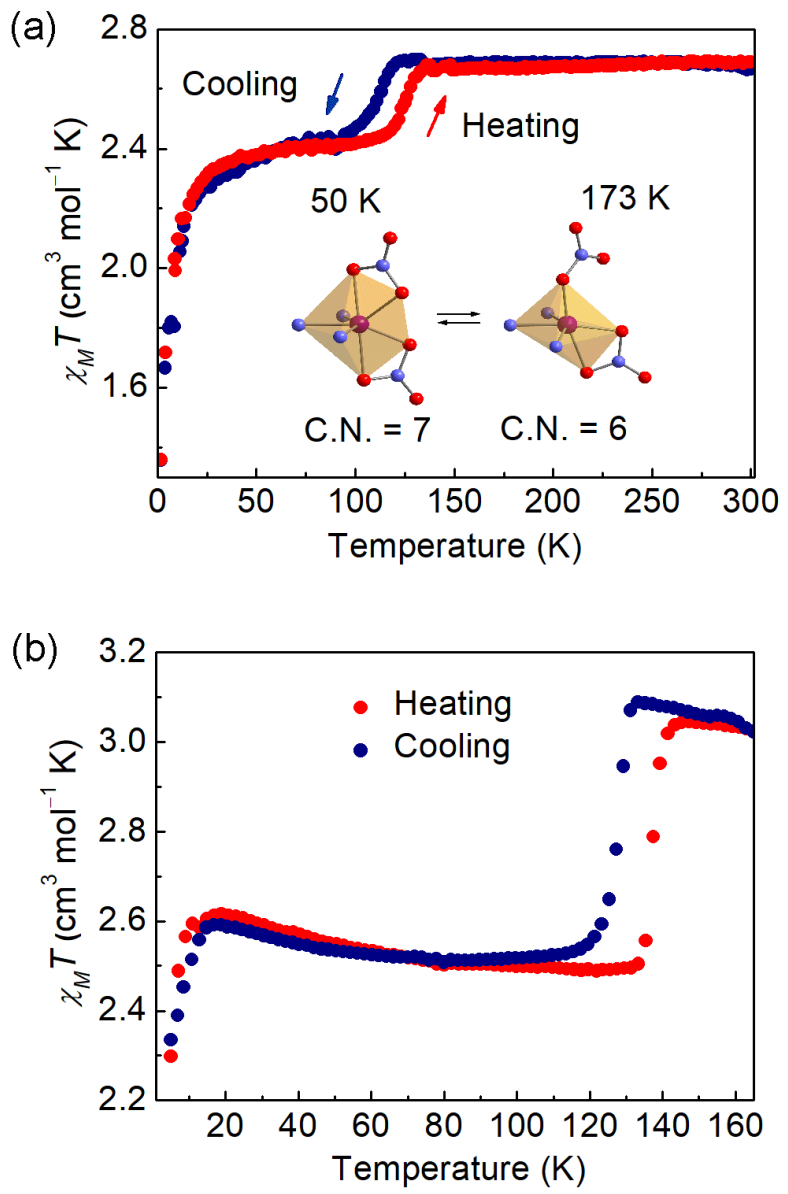

Figure 2. (a) Variable-temperature of $\chi_{\mathrm{M}} T$ for a microcrystalline sample of 1 exhibits an abrupt change at around $118 \mathrm{~K}$ with a hysteresis of $c a .14 \mathrm{~K}$. The $\chi_{\mathrm{M}} T$ value changes by about $10 \%$ after the transition. The inset shows the change of the coordination number (C.N.) and coordination geometry after the phase transition. Purple, Co; blue, N; red, O. (b) Temperature dependent $\chi_{\mathrm{M}} T$ along the easy axis for the oriented sample with $c a .20 \%$ variations. 
quenched $\left(1.88 \mathrm{~cm}^{3} \mathrm{~mol}^{-1} \mathrm{~K}\right)$ and unquenched $\left(5.49 \mathrm{~cm}^{3} \mathrm{~mol}^{-1} \mathrm{~K}\right)$ angular momentum. This indicates that a significant contribution of the angular momentum is present, as reflected in the $\mathrm{g}$ values, which are estimated from the Curie constants obtained by magnetic measurements to be $g=2.42$ and 2.31 in the highand low-temperature ranges, respectively. When the temperature is increased, the $\chi_{\mathrm{M}} T$ value abruptly increases at around 135 $\mathrm{K}$ with a hysteresis loop of $14 \mathrm{~K}$. This indicates that the change in the $\chi_{M} T$ value was caused by a first-order phase transition. The $\chi_{\mathrm{M}} T$ demonstrates an increment with an order of $10.0 \%$ $11.0 \%$ at around the transition point. Along the easy axis, the $\chi_{\mathrm{M}} T$ value varied by ca. $20 \%$, as measured on microcrystals oriented and frozen in toluene (Figure $2 b$ ). This change is more obvious and the result indicates large magnetic anisotropy in complex 1. As neither spin crossover nor valence tautomerism occurs and lack of dominant magnetic coupling $\left(\mathrm{d}_{\mathrm{co}_{0}-\mathrm{C}_{0}}>6.60 \AA\right.$ for the high-temperature phase), the change in magnetization should be a result of the modulation of the orbital angular momentum through the structural perturbation facilitated by the dynamic bond. ${ }^{2123}$ Furthermore, field-induced slow relaxation of the magnetization was observed in $\mathbf{1}$ (Figure S4).

Calculations. To explain our experimental observations and provide detailed insight into the change in magnetization, ab initio calculations using the CASSCF/NEVPT2 (Complete-Active-Space Self-Consistant-Field/N-Electron Perturbation Theory) protocol were performed starting with the crystallographically determined structures at low and high temperatures (Tables S6 and S7). The energy differences between two states is $6.4 \mathrm{kcal} \mathrm{mol}^{-1}$, which is in the reasonable region of that of switchable materials. The temperature dependence of the magnetic susceptibility $\chi_{\mathrm{M}} T$ obtained by quasi-degenerate perturbation theory agrees well with the experimental observation..$^{11^{132}}$ Projecting the calculated two low-lying doublets onto the spin Hamiltonian with $\mathrm{S}=3 / 2$ yields the following phenomenological magnetic parameters: $\mathrm{D}=-8.31 \mathrm{~cm}^{-1}, \mid \mathrm{El}=1.49 \mathrm{~cm}^{-1}$, and $g_{\text {so }}$ $=2.23$ for the low-temperature phase (where D and E are the axial and rhombic anisotropic parameters respectively; and $g_{\text {so }}$ is the isotropic $g$-factor); and $\mathrm{D}=-31.45 \mathrm{~cm}^{-1}, \mid \mathrm{El}=10.32 \mathrm{~cm}^{-1}$, and $g_{\text {io }}=2.33$ for the high-temperature phase (Figure 3a), which are in reasonable agreement with the parameters derived from the DC magnetic data (Figure S5). The observed large change in the magnetic moments is ascribed to the quenching of the orbital angular momentum in the LT phase. The orbital contributions to $g$-factors of the ground doublet also exhibit significant change; the ratio of isotropic orbital contribution and spin contribution $\left(g_{\nu} / g_{s}\right)$ decrease from 0.233 for HT phase to 0.134 for LT phase (Figure S6 and Table S8).

We further analyzed the electronic states of $\mathbf{1}$ in the LT and HT phases. Without inclusion of spin-orbit coupling (SOC), the ground states of both phases are orbital singlets and well separated from their first excited states $\left(3841 \mathrm{~cm}^{-1}\right.$ for LT and 1397 $\mathrm{cm}^{-1}$ for HT, respectively. Figure $3 \mathrm{~b}$ ) due to the low symmetry, and orbital contribution should be completely quenched. However, inclusion of SOC mixes excited state character into the ground state with complex coefficients. Therefore, the orbital angular momentum is partially recovered. The mixing coefficient of the excited wavefunction is inversely proportional to the energy gap between the excited states and the ground state (in the magnitude of $\zeta / \Delta \mathrm{E}$, where $\zeta$ is the SOC constant of $\mathrm{Co}(\mathrm{II})$ and $\Delta \mathrm{E}$ is the energy gap between the excited state and ground state), which leads to a greater recovery of the orbital contribution in the HT structure.
$\mathrm{Ab}$ initio ligand-field theory (AILFT), maps the CASSCF/NEVPT2 level results onto a ligand-field type Hamiltonian, thus providing unique information about the ligand-
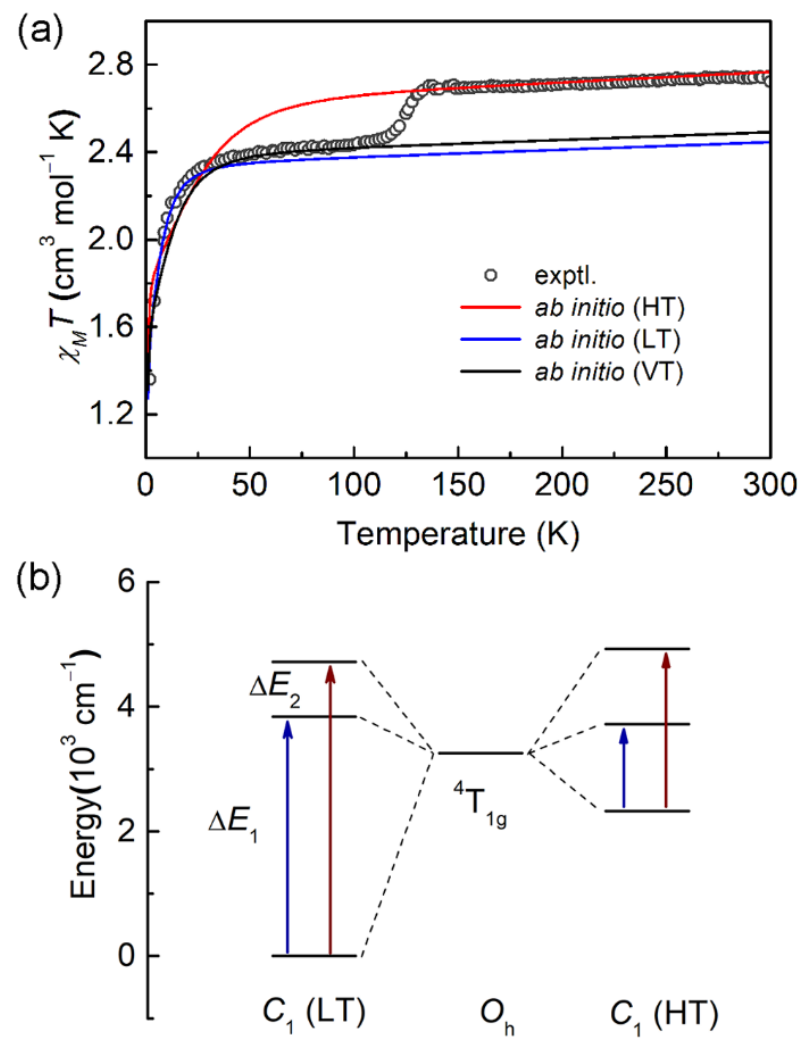

Figure 3. (a) $\chi_{\mathrm{M}} T$ curves for complex 1 and the virtual molecule obtained from $a b$ initio calculations. The calculated $\chi_{\mathrm{m}} T$ values in the LT and HT phases are both slightly underestimated. The changes are well consistent with the experimental data. The calculated $\chi_{\mathrm{M}} T$ value for the virtual state is very close to that of the LT phase. (b) Energy level diagram showing the splitting of the free ion quartet states under the influence of a ligand field with $C_{1}$ symmetry in the LT and HT phases. The arrows depict the transitions calculated.

field (including ligand-field splitting and Racah parameters, B and $\mathrm{C}$ ). This information affords the opportunity to understand electronic structure from a more intuitive chemical picture without oversimplification. Here we adopt this approach to understand the angular momentum switching behavior in this compound. The $\mathrm{Co}(\mathrm{II})$ ion in the ideal $\mathrm{O}_{n}$ field possesses three degenerate $t_{2 g}$ orbitals, determining a ground ${ }^{4} T_{1}$ term (Figure $S 7$ ). Being an orbital triplet, an unquenched $L_{\mathrm{cff}}=1$ should be expected. However, deviation from ideal $\mathrm{O}_{\mathrm{n}}$ symmetry in the HT 6-coordinate structure remove such orbital degeneracy, splitting the $\mathrm{t}_{28}$ orbitals by 0,198 and $865 \mathrm{~cm}^{-1}$ (Figure $4 \mathrm{a}$ and Figure $\mathrm{S} 8 \mathrm{a}$ ). This splitting is on the same order of magnitude as the SOC constant of a free $\mathrm{Co}(\mathrm{II})$ ion $\left(\zeta=533 \mathrm{~cm}^{-1}\right)$. The electronic structure can be approximately interpreted as the result of a trigonal distortion, which splits the $\mathrm{t}_{2 \mathrm{~g}}$ orbitals into two, $\mathrm{e}_{(12)}$ and $\mathrm{a}_{1(2) 2}$. Therefore, a significant unquenched orbital angular momentum is recovered in the ground state (Figure 4b). A seventh coordination along the $\mathrm{C}_{3}$-axis significantly enhances the energy gap between $\mathrm{e}_{(12)}$ and $\mathrm{a}_{1(12)}$ orbitals. Indeed, in the LT 7-coordinated form, the AILFT analysis gives two pseudo-degenerate d orbitals with the splitting energy of $508 \mathrm{~cm}^{-1}$, while the other d orbital lies at 3283 $\mathrm{cm}^{-1}$ above the lowest $\mathrm{d}$ orbital leading to a much larger energy 

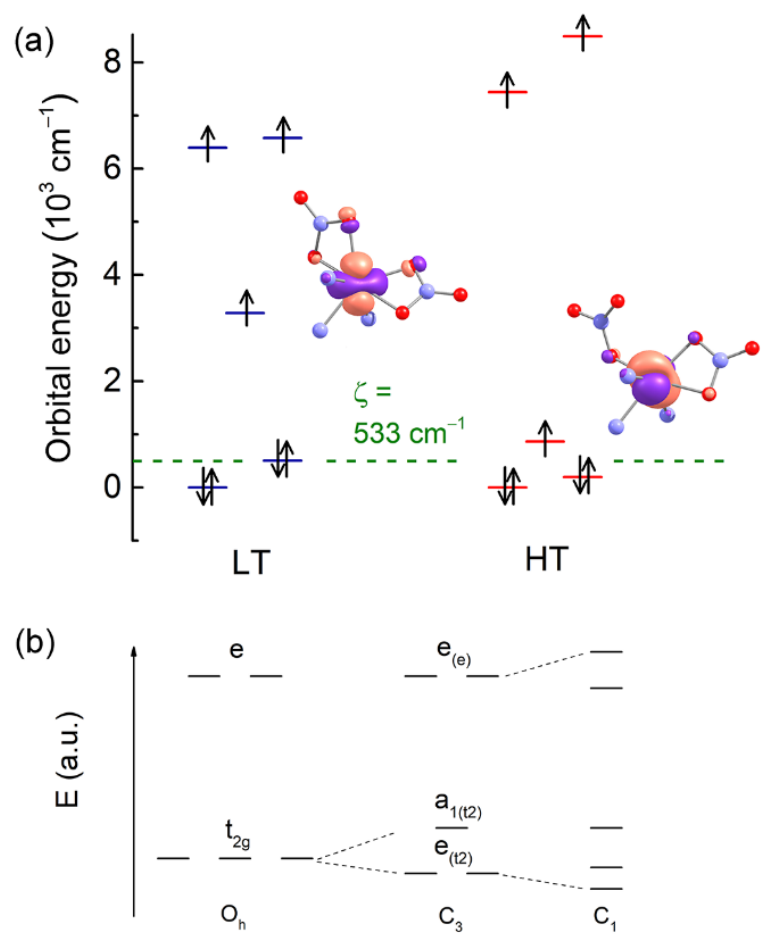

Figure 4. (a) AILFT orbital diagrams showing the calculated dorbital splitting in the LT phase (blue) and the HT phase (red). Horizontal lines depict orbital energies while arrows pointing up, or down stand for single electron spins. The green line represents the SOC constant $\zeta$ of free Co(II) ion. The first singly occupied $a b$ initio ligand-field orbitals are accompanied by representations of their respective molecular orbitals. In the low-temperature phase, evidence of covalent $\sigma^{*}$ character in the Co1-O dynamic bond is observed. Uncoordinated atoms from the ligand $\mathrm{L}$ are excluded for clarity. (b) The schematic d orbital splitting diagram for six-coordinated transition-metal complexes with $\mathrm{O}_{n}, \mathrm{C}_{3}$ and $\mathrm{C}_{1}$ symmetry.

splitting compared to the SOC constant. This change determines a well-defined orbital singlet as the ground ligand-field term; and induces an increase in the excited-state energies, which leads to a substantial quenching in the contribution of the orbital angular momentum. The calculated ligand field schemes for the 6- and 7-coordinated form are also consistent with the SOC-free electronic configuration energies; smaller energy gaps are found in the 6-coordinated form, while the ground state is well-separated from the other two excited states in the 7-coordinated form. . $^{3}$

X-ray absorption spectroscopy and X-ray Magnetic Circular Dichroism. To detect changes in the ligand field splitting at the d-orbitals in going from the 7-coordinate to 6-coordinate structure, X-ray absorption spectroscopy (XAS) at the Co $L_{23}-$ edge were measured at 5 and $150 \mathrm{~K}$ (Figure 5a). Clear changes are observed at the top of the $\mathrm{L}_{3}$-edge and a feature at $776.9 \mathrm{eV}$ gains intensity and shifts to lower energy by $0.1 \mathrm{eV}$ in going from LT to HT. Circularly polarized XAS measurements show strong dichroism in a saturating magnetic field of $14 \mathrm{~T}$ (Figure $5 b)$. Figures $5 c$ and $S 9$ shows the difference between the left and right circularly polarized spectra, the X-ray magnetic circular dichroism (XMCD). Crystal field multiplet calculations simulate the XAS and XMCD results. For the simulation of the 5 $\mathrm{K}$ results we approximate the symmetry of the 7-coordinate cluster to a $\mathrm{C}_{3 \mathrm{v}}$ point group. The crystal field is parameterized by three independent parameters, Dq, Do and $\mathrm{D} \tau .^{34}$ A positive $\mathrm{Dq}$ parameter splits the energy of $3 d$ orbitals into symmetry sets $t_{2}$ and $\mathrm{e}^{2}$. The inclusion of D $\sigma$ and $\mathrm{D} \tau$ parameters break the lower energy $t_{2}$ symmetry set into $e_{(2)}$ and $a_{1(2)}$. Simulations adjusting these parameters were performed. Reproduction of the experimental results was found to require a $\mathrm{e}_{(2)^{4}} \mathbf{a}_{1(2)}, \mathbf{e}_{(0)}{ }^{2}$ ground state configuration. The parameters for simulating the $5 \mathrm{~K}$ data in Fig. 5 are $\mathrm{Dq}=0.0775 \mathrm{eV}, \mathrm{D} \tau=-0.03 \mathrm{eV}$ and $\mathrm{D} \sigma=-0.065 \mathrm{eV}$, which split the crystal field evenly with a total energy between the e doublets of $0.8 \mathrm{eV}$. For the simulation of the $150 \mathrm{~K}$ data the $\mathrm{C}_{3 \mathrm{~s}}$ distortion introduced by the seventh coordination bond was relaxed, reducing the splitting between the $\mathrm{e}_{(2)}$ and $\mathrm{a}_{1(2)}$ orbitals,

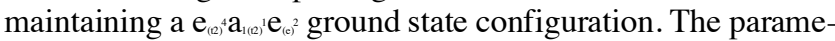
ters for simulating the $150 \mathrm{~K}$ data shown in Fig. $3 \mathrm{a}$ are $\mathrm{Dq}=0.1$ $\mathrm{eV}, \mathrm{D} \tau=-0.03 \mathrm{eV}$ and $\mathrm{D} \sigma=0.03 \mathrm{eV}$. The crystal field from these parameters give a smaller $\mathrm{e}_{(2)}-\mathrm{a}_{1(1)}$ splitting of just $0.12 \mathrm{eV}$ with a total splitting between e doublets of $0.96 \mathrm{eV}$. As shown in supplementary Fig. S8b, the obtained splitting parameters are consistent with the AILFT results. In addition, to fitting the measured results using a $\mathrm{C}_{3 \mathrm{v}}$ symmetry model, we took the AILFT CASSCF results and used the determined AILFT matrix

(a)

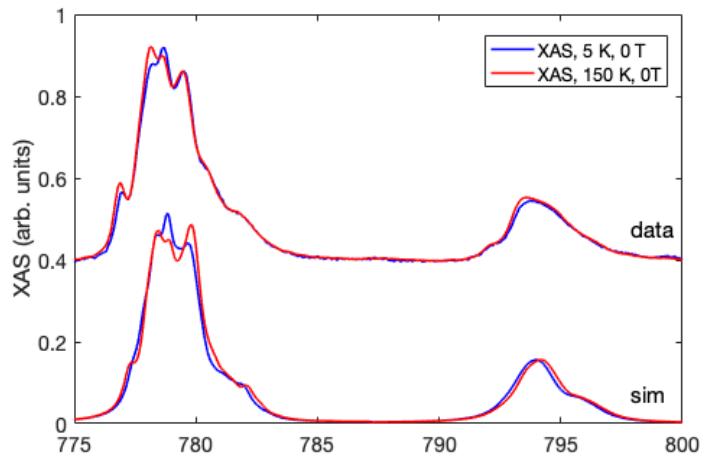

(b)

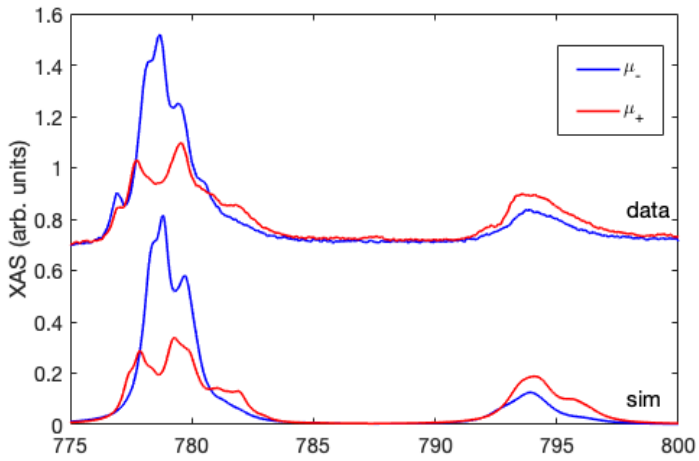

(c)

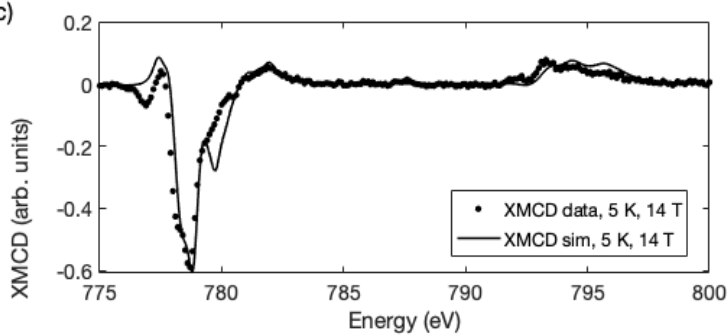

Figure 5. X-ray absorption and XMCD spectra at the Co $L_{23}$ edges for complex 1. (a) Measured (upper) and simulated (lower) $L_{23}$ edge XAS $\left(\mu_{+}+\mu_{\text {. }}\right) / 2$ at 5 and $150 \mathrm{~K}, 0 \mathrm{~T}$. (b) Measured (upper) and simulated (lower) circularly polarized XAS measured at $5 \mathrm{~K}$ and $14 \mathrm{~T}$. (c) Measured and simulated XMCD $\left(\mu_{+}-\mu\right.$ ) spectra, at $5 \mathrm{~K}$ and $14 \mathrm{~T}$. 
elements, Racah parameters and SOC parameter to perform further crystal field multiplet simulations (Figure S10). The simulated $L_{3} \mathrm{XMCD}$ fits the data very well. However, the simulated $L_{2}$ edge XMCD is too intense, this is an indication of the overestimation on spin-orbit quenching by CASSCF. The $5 \mathrm{~K}$ XAS spectrum lacks features in the $L_{3}$-edge suggesting under estimation in ligand field strength. The $150 \mathrm{~K} L$-edge XAS simulation is particularly improved. This is an interesting combination of $\mathrm{ab}$ initio and multiplet theory that is not often applied in the simulation of XAS and XMCD.

The effect of dynamic bond on magnetic switching. To correlate the dynamic bond with the magnetic changes rather than the other structural parameters, we calculated the electronic state of a virtual molecule, which was constructed by rotating the $\mathrm{NO}_{3}$ - anion around the $\mathrm{O} 1$ atom $\mathrm{ca} .25 .3^{\circ}$ in the hightemperature phase to form the Co1-O3 bond found in the lowtemperature phase (Figure S11). Apart from the $25.3^{\circ}$ rotation of the $\mathrm{NO}_{3}$ - anion, the structure of the virtual molecule is identical to that of the high-temperature phase. The calculated $\chi_{\mathrm{M}} T$ value is also close to that of the LT phase (Figure 3a). Moreover, the calculated $g_{/} / g_{s}$ value is 0.167 , also close to that of the LT phase. These results suggest that the dynamic Co1-O3 bond plays the crucial role in the magnetic changes. Analysis based on the $a b$ initio ligand field orbitals reveals that the strong antibonding interaction between the $\mathrm{d}$ orbitals centered on Co1 and the $\mathrm{O} 3$ from the nitrate group significantly raises the energy level of the lowest single-occupied d orbital (Figure 4 and Figure S8a), giving rise to the much larger two lowest excited energies compared with high-temperature structure.

We should note that ligand field strength and spin-paring energy compete in spin-crossover complexes. On the other hand, it is thought that the gain by the coordination bond formation and the loss by steric repulsion are important factors in dynamic bond systems undergoing a change in the coordination number from six to seven. The use of nitrate ligands allows stabilizing of the seven-coordinate structure because of the relatively weak steric effect owing to the small bite angle of the bidentate mode and the small size of the nitrate ligand, which leads to competition between the six- and seven-coordinate structure in $\mathbf{1}$. This consideration and the observation of the magnetization changes in 1 suggest that the combination of $\mathrm{Co}$ (II) and nitrate ligands would be appropriate to rationally realize the orbital angular momentum switching via dynamic bonds.

The change of magnetization observed here originates from the reversible quenching and restoration of the orbital angular momentum. This mechanism differs greatly from the preexisting ones, such as spin crossover between the high- and lowspin states of a transition-metal ion, ${ }^{36}$ charge transfer,,$^{12,15}$ spincrossover-coupled charge transfer, tions of the organic radicals, ${ }^{16-18}$ spin-Peierls-type transitions, and modification of magnetic coupling induced by a structural phase transition..$^{3.38}$

\section{CONCLUSIONS}

Orbital angular momentum plays an important role in the magnetic, optical, and conducting properties of functional materials. Control of the orbital angular momentum via modulation of the effect of relativistic spin-orbit coupling is thus of importance to switch these properties by external stimuli. Our strategy to induce a large change in the orbital angular momentum involved the induction of coordination mode switching of a nitrate in a crystalline $\mathrm{Co}$ (II) complex, which involves the transformation between the monodentate and bidentate coordination modes of the nitrate ligand. The dynamic bond, i.e. the breaking and re-formation of a coordination bond, induced the largest change in the orbital angular momentum of a molecular crystalline material reported to date. Notably, this technique of switching coordination number could be widely used to control various physical properties, not only orbital angular momentum.

\section{EXPERIMENTAL SECTION}

Magnetometry. DC magnetic susceptibility measurements were performed on an MPMS-5S SQUID magnetometer in an applied field of 5000 Oe over the entire temperature range. All data were corrected for diamagnetic contributions. To measure the magnetic susceptibility along the easy axis, the microcrystals were dispersed in toluene and field aligned in an applied magnetic field of $5 \mathrm{~T}$ at $300 \mathrm{~K}$. Cooling to $165 \mathrm{~K}$, frozen the field orientated crystals; subsequently, measurements commenced in a field of 5000 Oe. ${ }^{39}$

X-ray spectroscopy. XAS and XMCD measurements at the Co $L_{23}$ absorption edges were measured on beamline I10 at Diamond Light Source, Harwell Science and Innovation Campus, Oxfordshire, U.K. The XMCD spectra were obtained by flipping the helicity of circularly polarized X-rays exhibiting a $100 \%$ degree of polarization in a fixed applied magnetic field. The measurements were performed with the temperature of the sample holder being regulated between 4.5 and $300 \mathrm{~K}$. The total electron yield (TEY) was obtained by measuring the drain current of the sample. Orientation averaged polycrystalline samples of 1 were attached with carbon tape, to a copper sample holder. Radiolysis was controlled through the attenuation of the incident X-ray flux to $7 \%$ of the optimized value.

Crystal field multiplet simulations were performed using Quanty and Crispy in the $\mathrm{C}_{3 v}$ point group. The crystal field parameters $\mathrm{Dq}, \mathrm{D} \sigma$ and $\mathrm{D} \tau$ were obtained by fitting to the experimental $\mathrm{L}_{23}$-edge data. Atomic multiplet effects from electronelectron repulsions and exchange are parameterized using slater integrals calculated from atomic Hartree-Fock theory scaled down by a factor of $70 \%$ to account for the electronic delocalization of the $3 \mathrm{~d}$ electrons. ${ }^{\text {and }}$ The atomic spin-orbit coupling parameters on the $2 \mathrm{p}$ and $3 \mathrm{~d}$ open shells take values from the calculated spherical monoelectronic potential around the free ion $\left(\zeta_{3 \mathrm{st}}=0.066 \mathrm{eV}, \zeta_{\text {3is }}=0.083\right.$ and $\left.\zeta_{2 \mathrm{ps}}=9.7486 \mathrm{eV}\right)$. To reproduce core-hole broadening and instrumental resolution, a $0.5 \mathrm{eV}$ full width half maximum (FWHM) gaussian broadening is added to both edges and a Lorentzian broadening of 0.6 and $1.2 \mathrm{eV}$ are added to the $L_{3}$ and $L_{2}$-edge respectively.

An alternative method for simulating the XAS and XMCD results, free of adjustable parameters, was adopted in Fig. S10. The $3 \mathrm{~d}$ orbital crystal field matrix, B, C and $\zeta_{3 \mathrm{x}}$ are obtained directly from the AILFT CASSCF results. This removes the crystal field fitting parameters from the model. To account for multiplet effects present in the absorption final state, the Slater integrals determined by HF theory scaled to $70 \%$ are used. There are hence no free parameters other than the line width broadening for this model.

Computational methods. All ab initio calculations were performed with the ORCA program package Version 4.1.2.42,43 For all the calculations, the TZVPP basis with the corresponding auxiliary sets (def2-TZVPP/JK) were used. ${ }^{\text {tas }}$ The initial coordinates obtained high-temperature and low-temperature phase were employed for DFT calculations with constraints. Only the hydrogen atomic positions were optimized at the B3LYP/TZVP 
level of theory. ${ }^{6677}$ The Complete-Active-Space Self-Consistent Field (CASSCF) method was used to take the static correlation effect into account, while the N-Electron-Valence Perturbation Theory (NEVPT2) correction was adopted to consider the dynamic correlation effect. ${ }^{48}$ In the active space of CASSCF calculations 7 electrons in 5 atomic d orbitals $(\operatorname{CAS}(7,5))$ were employed. $10 \mathrm{~S}=3 / 2$ and $40 \mathrm{~S}=1 / 2$ states were taken into consideration. Spin-orbit coupling was taken into account using a mean-field spin-orbit coupling operator. The magnetic susceptibility was calculated by the quasi-degenerate perturbation theory in a 5000 Oe external field. The results were projected onto the ligand field scheme by the ab initio ligand field analysis implemented in the ORCA package ${ }^{3}$. To take the bonding effect into consideration, the active space was enlarged to CAS $(13,8)$ incorporating the bonding pairs of electrons. The calculated susceptibility was improved slightly, and the general chemical picture of the observed phenomena remained unchanged. The calculated results including the Mayer bond order and the diagram of natural bond orbitals are provided in the Table S5.

\section{ASSOCIATED CONTENT}

\section{Supporting Information}

The Supporting Information is available free of charge on the ACS Publications website at DOI:

Experimental conditions of synthesis, X-ray structural determination and heat capacity. Additional crystallographic figures and tables, PXRD, dynamic magnetic data, the calculated d-orbital splitting and the orbital contribution, and crystallographic information files (CIFs). CCDC 1489229 ( 1 at $50 \mathrm{~K}$ ) and 1489230 ( 1 at $173 \mathrm{~K}$ ) containing the supplementary crystallographic data can be obtained free of charge from the Cambridge Crystallographic Data Center via www.ccdc.cam.ac.uk/data_request/cif..

\section{AUTHOR INFORMATION}

\section{Corresponding Author \\ *sato@cm.kyushu-u.ac.jp}

\section{ACKNOWLEDGMENT}

This work was supported by JSPS KAKENHI Grant Number 17H01197. This work was performed under the Cooperative Research Program of "Network Joint Research Center for Materials and Devices. This work was supported by the MEXT Project of "Integrated Research Consortium on Chemical Sciences". $\mathrm{L}_{2,3-}$ edge XAS and XMCD experiments were carried out with the support of the Diamond Light Source.

\section{REFERENCES}

(1) Sato, O. Dynamic molecular crystals with switchable physical properties. Nature Chem. 2016, 8, 644-656.

(2) Sessoli, R.; Gatteschi, D.; Caneschi, A.; Novak, M. A. Magnetic bistability in a metal-ion cluster. Nature 1993, 365, 141 - 143.

(3) Halcrow, M. A. Spin-Crossover Materials: Properties and Applications. (Wiley, 2013).

(4) Hardinga, D. J.; Hardinga, P; Phonsrib, W. Spin crossover in iron(III) complexes. Coord. Chem. Rev. 2016, 313, 38-61.

(5) Bousseksou, A.; Molnár, G., Salmon, L.; Nicolazzi, W. Molecular spin crossover phenomenon: recent achievements and prospects. Chem. Soc. Rev. 2011, 40, 3313-3335.

(6) Gutlich, P.; Hauser, A.; Spiering, H. Thermal and optical switching of iron(II) complexes. Angew. Chem. Int. Ed. 1994, 33, 2024-2054.

(7) Tezgerevska, T.; Alley, K. G.; Boskovic, C. Valence tautomerism in metal complexes: Stimulated and reversible intramolecular electron transfer between metal centers and organic ligands. Coord. Chem. Rev. 2014, 268, 23-40.
(8) Nihei, M.; Okamoto, Y.; Sekine, Y.; Hoshino, N.; Shiga, T.; Liu, I. P.-C.; Oshio, H. A light-induced phase exhibiting slow magnetic relaxation in a cyanide-bridged $\mathrm{Fe}_{2} \mathrm{Co}_{2}$ complex. Angew. Chem. Int. Ed., 2012, 51, 6361-6364.

(9) Hilfiger, M. G.; Chen, M.; Brinzari, T. V.; Nocera, T. M.; Shatruk, M.; Petasis, D. T.; Musfeldt, J. L.; Achim, C.; Dunbar, K. R. An unprecedented charge transfer induced spin transition in an $\mathrm{Fe}-\mathrm{Os}$ cluster. Angew. Chem., Int. Ed. 2010, 49, 1410-1413.

(10) Ohkoshi, S.; Tokoro, H. Photomagnetism in cyano-bridged bimetal assemblies. Acc. Chem. Res., 2012, 45, 1749-1758.

(11) Sessoli, R. Magnetic clusters: Spinning into control. Nature Chem. 2010, 2, 346-347.

(12) Fedushkin, I. L.; Maslova, O. V.; Morozov, A. G.; Dechert, S.; Demeshko, S.; Meyer, F. Genuine redox isomerism in a rare-earthmetal complex. Angew. Chem. Int. Ed. 2012, 51, 10584-10587.

(13) Umezono, Y.; Fujita, W.; Awaga, K. Coordination bond formation at charge-transfer phase transition in (BDTA) $\left[\mathrm{Co}(\mathrm{mnt})_{2}\right] . J . A m$. Chem. Soc. 2006, 128, 1084-1085.

(14) Sergi Vela, M. D.; Ribas-Arino, J.; Novoa, J. J. Tracing the Sources of the Different Magnetic Behavior in the Two Phases of the Bistable (BDTA) $\left[\mathrm{Co}(\mathrm{mnt})_{2}\right]$ Compound. Inorg. Chem. 2012, 51, 8646-8648.

(15) Takaishi, S.; Ishihara, N.; Kubo, K.; Katoh, K.; Breedlove, B. K.; Miyasaka, H.; Yamashita, M. Paramagnetic diamagnetic phase transition accompanied by coordination bond formation dissociation in the dithiolate complex $\mathrm{Na}\left[\mathrm{Ni}(\mathrm{pdt})_{2}\right] \cdot 2 \mathrm{H}_{2} \mathrm{O}$. Inorg. Chem. 2011, 50, 6405-6407.

(16) Hicks, R. G. Switchable materials: A new spin on bistability. Nature Chem. 2011, 3, 189-191.

(17) Fujita, W.; Awaga, K. Room-iemperature magnetic bistability in organic radical crystals. Science 1999, 286, 261-262.

(18) Lekin, K.; Winter, S. M.; Downie, L. E.; Bao, X.; Tse, J. S.; Desgreniers, S.; Secco, R. A.; Dube, P. A.; Oakley, R. T. Hysteretic spin crossover between a bisdithiazolyl radical and Its hypervalent $\sigma$ dimer. J. Am. Chem. Soc. 2010, 132, 16212-16224.

(19) Craig, G. A.; Murrie, M. 3d single-ion magnets. Chem. Soc. Rev. 2015, 44, 2135-2147.

(20) Demir, S.; Jeon, I.-R.; Long, J. R.; Harris, T. D. Radical ligandcontaining single-molecule magnets. Coord. Chem. Rev. 2015, 289290, 149-176.

(21) Juhász, G.; Matsuda, R.; Kanegawa, S.; Inoue, K.; Sato, O.; Yoshizawa, K. Bistability of magnetization without spin-transition in a high-Spin cobalt(II) complex due to angular Momentum quenching. $J$. Am. Chem. Soc. 2009, 131, 4560-4561.

(22) Alley, K. G.; Poneti, G.; Robinson, P. S. D.; Nafady, A.; Moubaraki, B.; Aitken, J. B.; Drew, S. C.; Ritchie, C.; Abrahams, B. F.; Hocking, R. K.; Murray, K. S.; Bond, A. M.; Harris, H. H.; Sorace, L.; Boskovic, C.Redox activity and two-Step valence tautomerism in a family of dinuclear cobalt complexes with a spiroconjugated bis(dioxolene) ligand. J. Am. Chem. Soc. 2013, 135, 8304-8323.

(23) Su, S.-Q.; Kamachi, T.; Yao, Z.-S.; Huang, Y.-G.; Shiota, Y.; Yoshizawa, K.; Azuma, N.; Miyazaki, Y.; Nakano, M.; Maruta, G.; Takeda, S.; Kang, S.; Kanegawa, S.; Sato, O. Assembling an alkyl rotor to access abrupt and reversible crystalline deformation of a cobalt(II) complex. Nature Commun. 2015, 6, 8810 .

(24) Wojtecki, R. J.; Meador, M.; Rowan, S. J. Using the dynamic bond to access macroscopically responsive structurally dynamic polymers. Nature Mater. 2011, 10,14-27.

(25) Venkataramani, S.; Jana, U.; Dommaschk, M.; Sönnichsen, F. D.; Tuczek, F.; Herges, R. Magnetic bistability of molecules in homogeneous solution at room temperature. Science 2011, 331, 445-448.

(26) Thies, S.; Sell, H.; Sch€utt, C.; Bornholdt, C.; N€ather, C.; Tuczek, F.; Herges, R. Light-induced spin change by photodissociable external ligands: A new principle for magnetic switching of molecules. J. Am. Chem. Soc. 2011, 133, 16243-16250.

(27) Burnworth, M.; Tang, L.; Kumpfer, J. R.; Duncan, A. J.; Beyer, F. L.; Fiore, G. L.; Rowan, S. J.; Weder, C. Optically healable supramolecular polymers. Nature 2011, 472, 334-337.

(28) Seo, J.; Bonneau, C.; Matsuda, R.; Takata, M.; Kitagawa, S. Soft secondary building unit: Dynamic bond rearrangement on multinuclear 
core of porous coordination polymers in gas media. J. Am. Chem. Soc. 2011, 133, 9005-9013.

(29) Burger, B.; Dechert, S.; Große, C.; Demeshko, S.; Meyer, F. Visualising the carboxylate shift at a bioinspired diiron(II) site in the solid state. Chem. Commun. 2011, 47, 10428-10430.

(30) Kume, Y.; Miyazaki, Y.; Matsuo, T.; Suga, H. Low-temperature heat-capacities of ammonium hexachlorotellurate and its deuterated analog. J. Phys. Chem. Solids 1992, 53, 1297-1304.

(31) Rechkemmer, Y.; Breitgoff, F. D.; Meer, M. v. d.; Atanasov, M.; Hakl, M.; Orlita, M.; Neugebauer, P.; Neese, F.; Sarkar, B.; Slageren, J. v. A four-coordinate cobalt(II)single-ion magnet with coercivity and a very high energy barrier, Nature Commun., 2015, 7, 10467.

(32) Novikov, V. V.; Pavlov, A. A.; Nelyubina, Y. V.; Boulon, M.E.; Varzatskii, O. A.; Voloshin, Y. Z.; Winpenny, R. E. P. A Trigonal prismatic mononuclear cobalt(II) complex showing single-molecule magnet behavior. J. Am. Chem. Soc., 2015, 137, 9792-9795.

(33) Bar, A. K.; Pichon, C.; Sutter, J.-P. Magnetic anisotropy in twoto eight-coordinated transition-metal complexes: Recent developments in molecular magnetism. Coord. Chem. Rev. 2016, 308, 346-380.

(34) Koenig, E. Ligand Field Energy Diagrams, 1977, page 20-21, DOI:10.1007/978-1-4757-1529-3.

(35) Jeannin, O.; Clérac, R.; Fourmigué, M. Order-disorder iransition coupled with magnetic bistability in the ferricinium salt of a radical nickel dithiolene complex. J. Am. Chem. Soc. 2006, 128, 14649-14656.

(36) Leibeling, G.; Demeshko, S.; Dechert, S.; Meyer. F. Hysteretic magnetic bistability based on a molecular azide switch. Angew. Chem. Int. Ed. 2005, 44, 7111-7114.

(37) Burger, B.; Demeshko, S.; Bill, E.; Dechert, S.; Meyer, F. The carboxylate twist: Hysteretic bistability of a high-spin diiron(II) complex identified by mössbauer spectroscopy. Angew. Chem. Int. Ed. 2012, 51, $10045-10049$.
(38) Zhao, X.-H.; Huang, X. C.; Zhang, S. L.; Shao, D.; Wei, H. Y.; Wang, X. Y. Cation-dependent magnetic ordering and room-temperature bistability in azido-bridged perovskite-type compounds. J. Am. Chem. Soc. 2013, 135, 16006-16009.

(39) Friedman, J. R.; Sarachik, M. P. Macroscopic Measurement of Resonant Magnetization Tunneling in High-Spin Molecules. Phys. Rev. Lett. 1996, 76, 3830-3833.

(40) Haverkort, M. W.; M. Zwierzcki, M.; Andersen, O. K. Multiplet ligand-field theory using Wannier orbitals. Phys. Rev. B 2012, 85, 165113 .

(41) Retegan, M. Crispy: v0.7.3, DOI:10.5281/zenodo.3258065, 2019.

(42) Neese, F. The ORCA program system. Wiley Interdiscip. Rev. Comp. Mol. Sci. 2012, 2, 73-78.

(43) Neese, F. Software update: the ORCA program system, version 4.0. Wiley Interdiscip. Rev. Comp. Mol. Sci. 2017, 8, e1327.

(44) Weigend, F.; Ahlrichs, R. Balanced basis sets of split valence, triple zeta valence and quadruple zeta valence quality for $\mathrm{H}$ to $\mathrm{Rn}$ : Design and assessment of accuracy. Phys. Chem. Chem. Phys. 2005, 7, 3297-3305.

(45) Weigend, F. Hartree-Fock exchange fitting basis sets for $\mathrm{H}$ to Rn. J. Comput. Chem. 2008, 29, 167-175.

(46) Becke, A. D. Density-functional thermochemistry. III. The role of exact exchange. J. Chem. Phys. 1993, 98, 5648-5652.

(47) Lee, C.; Yang, W.; Parr, R. G. Development of the Colle-Salvetti correlation-energy formula into a functional of the electron density. Phys. Rev. B 1988, 37 785-789.

(48) Angeli, C.; Cimiraglia, R.; Evangelisti, S.; Leininger, T.; Malrieu, J.-P. Introduction of n-electron valence states for multireference perturbation theory. J. Chem. Phys. 2001, 114, 10252.

(49) Schweinfurth, D.; Sommer, M. G.; Atanasov, M.; Demeshko, S.; Hohloch, S.; Meyer, F.; Neese, F.; Sarkar, B. The ligand field of the azido ligand: insights into bonding parameters and magnetic anisotropy in a Co(II)-Azido complex. J. Am. Chem. Soc. 2015, 137, 1993-2005. 


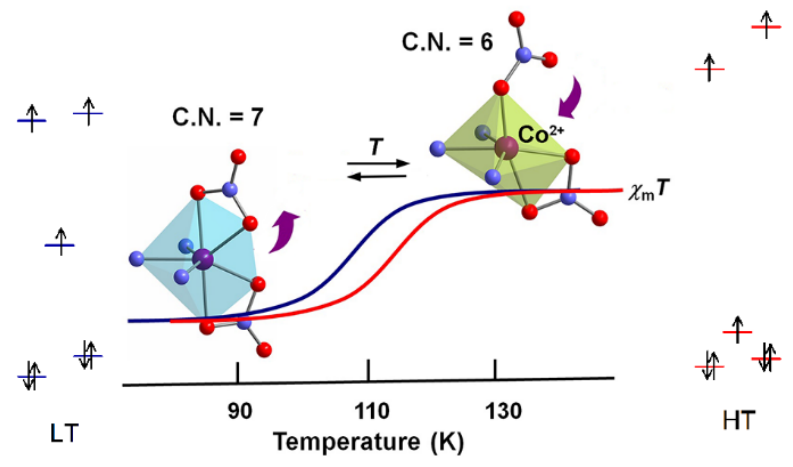

a graphic for the TOC 